\title{
Estado de pastos marinos en dos playas de la cayería norte, antes y después del paso del huracán Irma por la provincia de Ciego de Ávila, Cuba
}

\section{State of seagrass in two Northern Key beaches, before and after hurricane Irma passing through the Ciego de Ávila province, Cuba}

\author{
Claudia M. Cruz Pérez ${ }^{1 *}$, Claudia Bustamante López y Leslie Hernández Fernández ${ }^{2}$
}

\section{RESUMEN}

Los pastos marinos están considerados entre los ecosistemas más productivos de la biosfera. En la cayería norte de la provincia de Ciego de Ávila presentaron daños en su estructura y composición debido a la incidencia del huracán Irma. Luego del paso de este fenómeno, se evaluó el estado de los pastos marinos en playas Las Coloradas y El Paso, en noviembre de 2017, sobre la base de una caracterización realizada en junio de 2017. Para ambos muestreos, se ubicaron tres transectos de $50 \mathrm{~m}$ paralelos a la costa; en cada uno de ellos se situaron 12 marcos de $0.25 \mathrm{~m}^{2}$. La angiosperma marina predominante fue $T$. testudinum, en menor proporción S. filiforme. Se identificaron 25 especies de macroalgas y 4 de corales pétreos. Entre los invertebrados predominaron las especies $O$. reticulatus y L. gigas. Los peces de mayor abundancia fueron $H$. bivittatus y G. cinereus. En noviembre desapareció $S$. filiforme, disminuyó la diversidad de invertebrados y no se registró ictiofauna. El huracán Irma fue un evento meteorológico extremo que cambió la composición y estructura de las praderas de pastos marinos en los sitios de playa Las Coloradas y El Paso, al incidir en la disminución de la abundancia relativa y la densidad de vástagos de T. testudinum y afectar la altura del dosel y el porcentaje de epifitismo de esta especie, así como en la reducción de la fauna asociada. Se debe continuar el monitoreo de los sitios de pastos marinos, con el fin de estudiar su nivel de recuperación en el tiempo.

Palabras clave: Thalassia testudinum, Syringodium filiforme, huracán, fauna, archipiélago Sabana-Camagüey

\section{ABSTRACT}

Seagrass meadows are considered one of the most valuable ecosystems of the biosphere. In the Northern Keys of the Ciego de Ávila province, seagrass showed structural and composition

1 Centro de Investigaciones de Ecosistemas Costeros (CIEC), Avenida de los Almácigos s/n. Cayo Coco, Morón. Ciego de Ávila, Cuba. ccruz950929@gmail.com*, algas@ciec.cu

2 Departamento de Turismo y Organización Empresarial. Universidad “Máximo Gómez Báez”. Ciego de Ávila. Cuba. coraleslhf@gmail.com 
damages due to hurricane Irma's impact. The state of seagrass meadows was evaluated at Las Coloradas and El Paso beaches in November 2017 after the hurricane, based on a characterization conducted in June 2017. For both samples, three $50 \mathrm{~m}$ transects were located parallel to the coast. Along each transect, $120.25 \mathrm{~m}^{2}$ frames were placed. The predominant marine angiosperm was $T$. testudinum, and $S$. filiforme at a lower scale. A total of 25 macroalgae species and four stony coral species were identified. The most predominant invertebrate species found were $O$. reticulatus and L. gigas, while the most abundant fish species were H. bivittatus and G. cinereus. In November, $S$. filiforme disappeared, the diversity of invertebrates associated with seagrasses declined, and no ichthyofauna was recorded. Hurricane Irma was an extreme meteorological event that changed the composition and structure of seagrass meadows at Las Coloradas and El Paso beaches, because of its impact in the decrease of the relative abundance and shoot density of T. testudinum, the height of dossal, and the epiphytism percentage of the species, as well as the decrease in seagrass fauna. Seagrass sites must continue to be monitored to study their level of recovery over time.

Keywords: Thalassia testudinum, Syringodium filiforme, hurricane, fauna, Sabana-Camagüey Archipelago

\section{INTRODUCCIÓN}

Dentro de las hipótesis sobre el surgimiento de los pastos marinos, está aquella que plantea que estos se derivaron de plantas terrestres, costeras o dulceacuícolas que pasaron al mar de forma gradual (den Hartog \& Kuo, 2006). Los pastos marinos en Cuba dominan aproximadamente el $50 \%$ de los fondos con sedimentos particulados (arenosos o fangosos), y su distribución es discontinua, con una densidad variable en toda la plataforma marina (Vales et al. 1998). En el nivel del Caribe, constituyen uno de los ecosistemas más característicos e importantes de las zonas costeras (Rodríguez-Ramírez et al. 2005).

Los pastos marinos están considerados entre los ecosistemas más pro- ductivos de la biosfera (Herminga \& Duarte, 2000). En ellos habitan especies de casi todos los Phyla conocidos en el mar (Spalding et al. 2003). Contribuyen a la calidad del medio marino al amortiguar el oleaje y favorecen la retención de partículas suspendidas en el agua. Los pastos marinos y las macroalgas asociadas absorben nutrientes inorgánicos que mejoran la transparencia de la columna de agua, al actuar como filtros (Borum et al. 2004). Además, participan en la estabilización de los sedimentos y de la línea costera (Martínez-Daranas, 2007) lo que garantiza importantes servicios ambientales para el ser humano (Nordlund et al. 2018).

La humanidad obtiene bienes de los pastos marinos como los alimentos y productos de la industria cosmetoló- 
gica y médico-farmacológica (Martínez-Daranas, 2014), por sus propiedades antiinflamatorias, citoprotectoras, antioxidantes y neuroprotectoras (de la Torre-Nuñez et al. 2012). Además, estos ecosistemas, junto con los manglares y las crestas de arrecife, contribuyen a la protección de los asentamientos de personas en la zona costera, contra el oleaje provocado por los eventos meteorológicos extremos (Martínez-Daranas, 2010). A través de estos bienes y servicios, los pastos marinos favorecen la adaptación de la especie humana y la biodiversidad a los efectos del cambio climático (IPCC, 2007).

La dinámica de las praderas marinas que se desarrollan en los trópicos en ocasiones es afectada por el paso de tormentas y huracanes, los cuales provocan pérdidas en su biomasa foliar. La turbulencia generada por el tránsito de estos organismos trae consigo la redistribución de los sedimentos, la cual afecta a las praderas ya que pueden quedar enterradas o erosionadas según ocurra el desplazamiento de los sedimentos (Duarte \& Sand-Jensen, 1990; Marbà \& Duarte, 1994). Otros estudios también exponen sobre pérdidas de pastos marinos por desenterramiento o enterramientos ocasionados por fuerzas físicas (Ridler et al. 2006; van Tussenbroek et al. 2008).

En septiembre de 2017 ocurrió un evento meteorológico extremo, el huracán Irma (de categoría 5 en la escala Saffir-Simpson), que afectó la región del Caribe desde algunas islas de las Antillas Menores hasta los Estados Unidos pasando por Puerto Rico, República Dominicana, Haití y Cuba. El huracán Irma penetró a Cuba por las inmediaciones de la cayería norte en los límites entre las provincias de Camagüey y Ciego de Ávila, al este de Cayo Romano (Fig. 1). Dicho evento provocó vientos con velocidades que superaron los $100 \mathrm{~km} / \mathrm{h}$ durante ocho horas, con rachas máximas de $194 \mathrm{~km} / \mathrm{h}$ en Cayo Coco. Las olas en los cayos Coco y Guillermo llegaron a alcanzar alturas entre 4 y 7 m (Estación Meteorológica de primer orden 78 339, Centro de Investigaciones de Ecosistemas Costeros, CIEC).

El huracán Irma afectó toda la zona costera del Archipiélago Sabana-Camagüey (ASC), inclusive los ecosistemas marino-costeros de la cayería norte de la provincia de Ciego de Ávila (Estación Meteorológica de primer orden 78 339, CIEC), en cuya zona existe un alto desarrollo turístico. En dicha región, la zona costera es vulnerable a la acción antrópica debido a la afluencia del turismo y los efectos de los eventos meteorológicos (Batista-Tamayo et al. 2006). En esta región no se han realizado estudios con anterioridad para comprobar las perturbaciones que producen los eventos meteorológicos extremos sobre los ecosistemas marinos, específicamente sobre el de pastos marinos. 


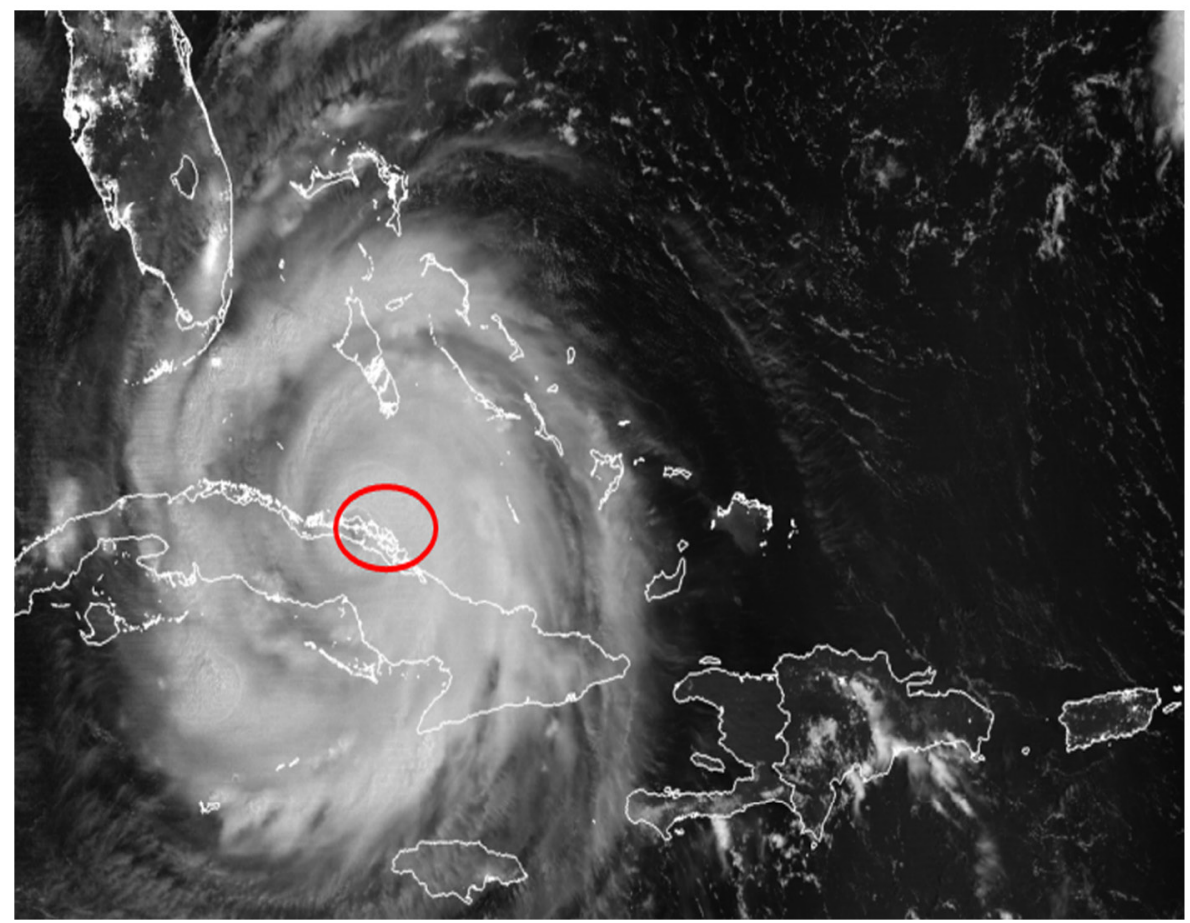

Fig. 1. Huracán Irma a su paso por la cayería norte de las provincias de Camagüey y Ciego de Ávila (9 septiembre, 2017). Fuente: https://cimss.ssec.wisc.edu/goes/ blog/archives/25001. Se representan los límites aproximados de las provincias de Camagüey y Ciego de Ávila y la ubicación de Cayo Coco y Cayo Romano. El círculo indica el centro del huracán a su paso por las inmediaciones de Cayo Coco Fig. 1. Hurricane Irma passing through the Northern Keys in the Camagüey and Ciego de Ávila provinces (September $9^{\text {th }}$, 2017). Source: https://cimss.ssec. wisc.edu/goes/blog/archives/25001. The approximate limit of the Camagüey and Ciego de Ávila provinces and the location of Cayo Coco and Cayo Romano are represented here. The circle shows the hurricane center as it passes through Cayo Coco

En la zona de estudio se tiene referencia de una investigación realizada a finales de la década de los noventa en un sitio ubicado en Cayo Coco, donde se empleó la metodología
CARICOMP (Alcolado et al. 1998), la cual abarcó aspectos sobre diversidad y cobertura. Además, hay registros de un análisis realizado por Clero-Alonso et al. (2006) sobre una caracterización 
general en algunos pastos marinos de los cayos Coco y Guillermo, y más recientemente, otro acerca de la caracterización realizada por Bustamante-López et al. (2016) a los pastos marinos en Cayo Paredón Grande.

De acuerdo con lo anterior, se asumió que los pastos marinos en la cayería norte de la provincia de Ciego de Ávila presentaron daños en su estructura y composición debido a la incidencia del huracán Irma. Con base en esta hipótesis, se escogieron dos de las playas de mayor calidad y atractivo turístico de la región (D. González-Alfonso \& O. de la Paz-Conde, comunicación personal, 31 de marzo del 2017) para evaluar el efecto del evento sobre los pastos marinos.

\section{MATERIALES Y MÉTODOS}

Área de estudio: Los sitios de estudio se encuentran localizados en dos playas, en los cayos Coco y Guillermo; playa Las Coloradas (22 $32^{\prime}$ $2.4^{\prime \prime}$ N $-78^{\circ} 20^{\prime} 38.4^{\prime \prime}$ 'W) y El Paso $\left(22^{\circ} 35^{\prime} 8.3^{\prime}, \mathrm{N}-78^{\circ} 38^{\prime}\right.$ 57.6' $\left.\mathrm{W}\right)$ (Fig. 2). Ambos cayos se encuentran ubicados al norte de la isla de Cuba en el ASC, específicamente, en el destino turístico Jardines del Rey. Cayo Coco se localiza a $30 \mathrm{~km}$ al noreste del pueblo de Punta Alegre, Ciego de Ávila, y abarca un área de $370 \mathrm{~km}^{2}$ con un largo de $31 \mathrm{~km}$. Cayo Guillermo se sitúa a $6 \mathrm{~km}$ al noroeste de Cayo Coco y ocupa un área de $13 \mathrm{~km}^{2}$ (Valdés-Montero et al. 2000). Según Batista-Tamayo et al. (2006), playa Las Coloradas tiene una pendiente fuerte, inclinada y la cual se profundiza muy cerca de la línea de costa; playa El Paso es todo lo contrario, tiene una pendiente suave, que se alarga y que provoca que las olas rompan lejos de la línea de costa. Ambas playas presentan un largo de la franja costera similar, para Playa Las Coloradas es de $2.8 \mathrm{~km}$ y para El Paso es de $2.5 \mathrm{~km}$, aproximadamente (Valdés-Montero et al. 2000).

\section{Diseño y método de muestreo:}

Los muestreos de los pastos marinos se realizaron en 2017, con una frecuencia de dos veces al año: en junio (antes del paso del huracán Irma) y en noviembre (después de este), teniendo en cuenta las variaciones espacio-temporales de las praderas de pastos marinos (junio; época lluviosa y noviembre; época poco lluviosa).

Para la descripción de la composición y estructura de los pastos marinos, se siguió la metodología propuesta por Martínez-Daranas et al. (2013) en el Protocolo para el monitoreo de los pastos marinos del proyecto "Aplicación de un enfoque regional al manejo de áreas costeras y marinas protegidas en los archipiélagos del sur de Cuba" (GEF/PNUD) N. ${ }^{\circ}$ 3973. Los dos sitios de playas fueron referenciados con GPS. Las áreas de muestreo fueron monitoreadas con un marco 

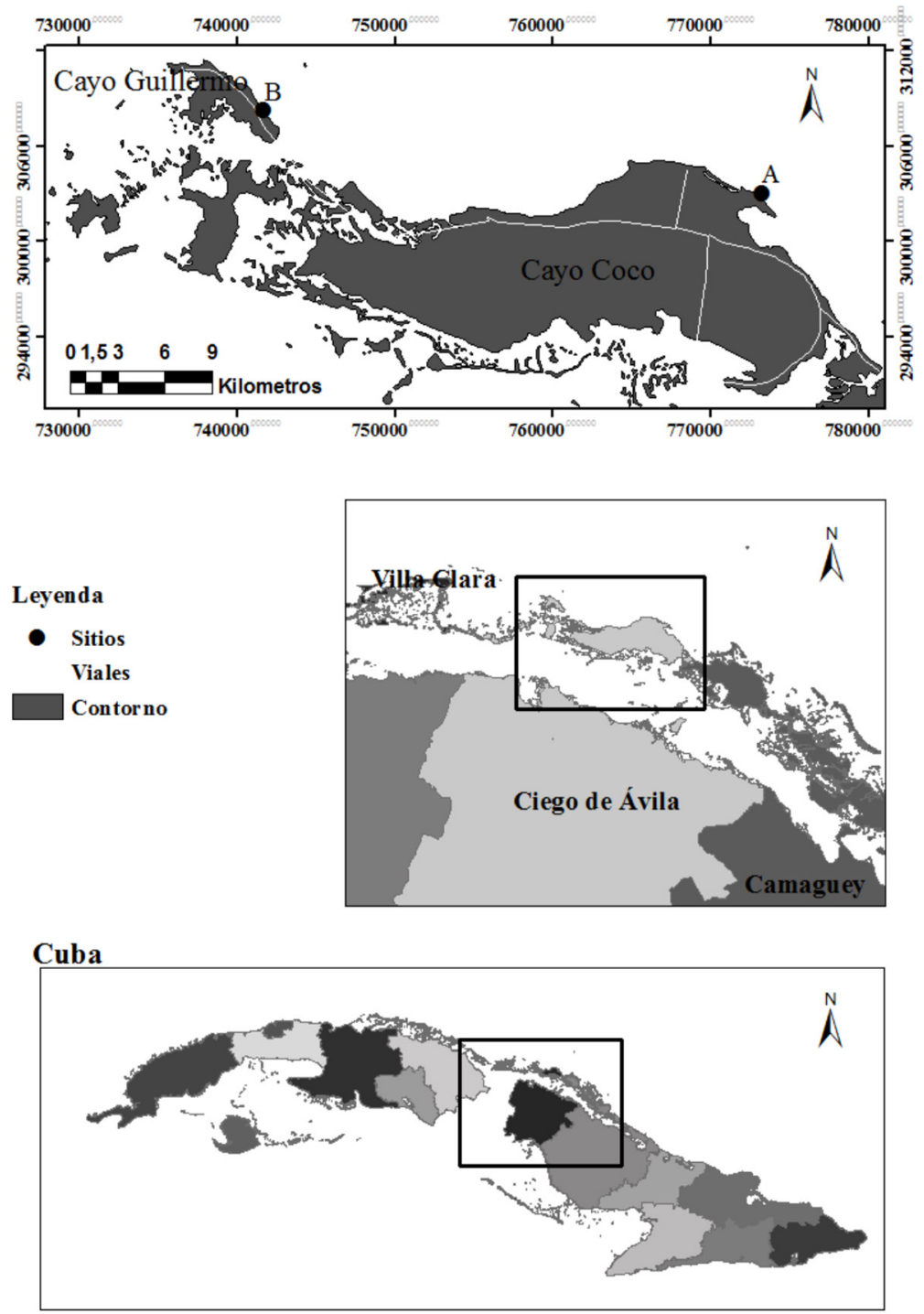

Fig. 2. Sitios de estudio de los pastos marinos, cayería norte, Ciego de Ávila. Playa Las Coloradas (A) y El Paso (B)

Fig. 2. Study sites in the seagrass meadows at the Northern Keys, Ciego de Ávila, Cuba. Las Coloradas beach (A) and El Paso beach (B) 
de $0.25 \mathrm{~m}^{2}$, colocado cada $4 \mathrm{~m}$ sobre tres transectos de $50 \mathrm{~m}$ paralelos a la costa, para un total de 12 marcos por transecto. Se colocó un transecto a cada extremo de la pradera a una distancia de $1 \mathrm{~m}$, aproximadamente de ambos bordes y otro intermedio entre los dos anteriores. Cada uno de ellos se ubicó teniendo en cuenta la extensión de la pradera.

En cada marco se identificaron las especies de angiospermas y la abundancia relativa (porcentaje de cobertura) por cada especie; se estimó la densidad de vástagos, la altura promedio de la vegetación (altura del dosel), así como el grado de epifitismo sobre las hojas de angiospermas. Además, se registró la presencia o ausencia de flores y frutos, la abundancia relativa de los grupos morfo-funcionales (GMF) (verdes calcáreas, carnosas, foliosas, globosas, filamentosas y costrosas) de macroalgas dominantes y la presencia de anomalías como la pradera erosionada o desenterramiento de los rizomas.

Las especies de macroalgas que no se lograron identificar in situ, se conservaron en frascos de cristal y se anotaron en una tablilla al momento de la colecta hasta su traslado al laboratorio donde fueron identificadas. Se clasificaron hasta el taxón más bajo posible, según criterios de Littler \& Littler (2000), Littler et al. (2008), Dawes \& Mathieson (2008), Suárez et al. (2015) y Guiry \& Guiry (2018). La profundidad y visibilidad horizontal fueron medidas in situ una sola vez por sitio en cada época de muestreo mediante distancia vertical y horizontal de disco Secchi, respectivamente.

\section{Fauna asociada a los pastos marinos}

Para la identificación de la fauna asociada a los pastos marinos en cada uno de los marcos, se determinó la presencia de invertebrados, en particular de corales pétreos. Para su clasificación, se siguieron los criterios de González-Ferrer (2009); en cada cuadrado, se contaron los reclutas de coral. Para la identificación de otros invertebrados, se siguieron los criterios de Humann (2002). También se registró la composición de peces sobre la base de lo establecido, por el método de censo visual (Brock, 1954) al seguir las modificaciones $y$ estaciones previamente inventariadas por Claro \& García-Arteaga (1994) y Claro et al. (2000). Para el estudio de las comunidades de peces, se utilizó el método de transecto lineal para especies seleccionadas. Se realizaron seis transectos de $30 \mathrm{~m}$ de longitud y $4 \mathrm{~m}$ de ancho. Los peces fueron clasificados hasta el taxón más bajo posible a partir de los criterios de Humann \& Deloach (2006).

\section{Análisis de datos}

Para determinar si existían diferencias entre los sitios en la estructura y composición de los pastos marinos, así como para conocer los efectos ocasionados por el paso del huracán Irma, se realizó un análisis 
de varianza (ANOVA) bifactorial, con un nivel de significación de 0.05 , con intervalos de confianza al $95 \%$, en el que se consideró como factor uno (fijo) los sitios (A: Las Coloradas y B: El Paso): y como factor dos (aleatorio) los meses (junio y noviembre), conscientes de que la dinámica en cada pradera fue inestable debido a la ubicación de las playas. Se utilizaron como variables la abundancia y la densidad de Thalassia testudinum Banks ex König, la altura promedio de las hojas y el porcentaje de epifitismo, en razón de que las praderas de pastos marinos estudiadas están conformadas mayormente por esta especie. Los gráficos y cálculos de análisis de varianza se ejecutaron con el programa STATISTICA 7.0 (StatSoft, 2004).

\section{RESULTADOS}

\section{Composición y estructura de los pastos marinos}

El sustrato predominante en las praderas de pastos marinos fue del tipo arenoso. La visibilidad horizontal en junio para las playas Las Coloradas y El Paso fue de 10 y 12 m, respectivamente, mientras que para noviembre disminuyó a 3 y $1 \mathrm{~m}$, correspondientemente. La profundidad aumentó para playa Las Coloradas de $0.6 \mathrm{~m}$ en junio a $1.6 \mathrm{~m}$ en noviembre, mientras que en playa El Paso disminuyó de $2.1 \mathrm{~m}$ en junio a $1.8 \mathrm{~m}$ en noviembre.
De forma general, al tomar en cuenta los resultados de junio y noviembre, la angiosperma marina predominante fue $T$. testudinum, mientras que en menor cobertura se observó a Syringodium filiforme Kütz. El desarrollo de pastos mixtos solo fue observado en playa El Paso (dos angiospermas marinas), mientras que el monoespecífico de $T$. testudinum en playa Las Coloradas. Del total ocupado por el macrofitobentos, en ambos sitios de muestreo, el 34.9\% de la superficie correspondió a pastos monoespecíficos de T. testudinum, el $22.1 \%$ a pastos mixtos y el $4.9 \%$ a parches de arena con macroalgas de los géneros Halimeda, Penicillus y Udotea, principalmente.

Enjunio, en playa Las Coloradas, el $40 \%$ de la superficie ocupada por el macrofitobentos correspondió a pastos monoespecíficos de $T$. testudinum, mientras que para noviembre afectó solo a un $29.7 \%$. La superficie ocupada por los pastos mixtos en playa El Paso estuvo representada por un $18.9 \%$ en junio, mientras que para noviembre desapareció $S$. filiforme lo que provocó un cambio de composición en la pradera, quedando conformada solamente por T. testudinum. No se observó presencia de flores o frutos en ninguno de los sitios en los meses muestreados. La pradera en ambas playas para noviembre se encontró erosionada con rizomas expuestos de T. testudinum. 
Tanto en junio como en noviemmostró una abundancia relativa de T. testudinum mayor que en El Paso. En este último sitio, la pradera estuvo conformada por un $15 \% \pm 10 \%$ y $4 \%$ $\pm 4.6 \%$ de $T$. testudinum y $S$. filiforme, respectivamente. En noviembre, también el sitio Las Coloradas mostró una abundancia relativa de $T$. testudinum mayor que El Paso. La cual mostró diferencias significativas entre meses $(\mathrm{F}=10.433 ; P<0.05)$ y entre sitios ( $\mathrm{F}=101.453 ; P<0.05)$, pero no entre sitios por meses $(\mathrm{F}=2.502 ; P>0.05)$ (Fig. 3A). bre, la densidad de T. testudinum fue mayor para el sitio Las Coloradas. Para junio, en el Paso, S. filiforme presentó una densidad de $80 \pm 90.3$ vástagos $\mathrm{m}^{-2}$. La densidad de vástagos disminuyó para ambos sitios en noviembre, $726.1 \pm 222$ vástagos $\mathrm{m}^{-2}$ para Las Coloradas y $205.3 \pm 58.8$ vástagos $\mathrm{m}^{-2}$ para el Paso. La densidad de vástagos según la prueba de ANOVA bifactorial mostró diferencias significativas entre meses $(\mathrm{F}=8.021 ; P<0.05)$ y entre sitios $(\mathrm{F}=312.831 ; P<0.05)$, pero no entre sitios por meses $(\mathrm{F}=0.871 ; P>0.05)$ (Fig. 3B).
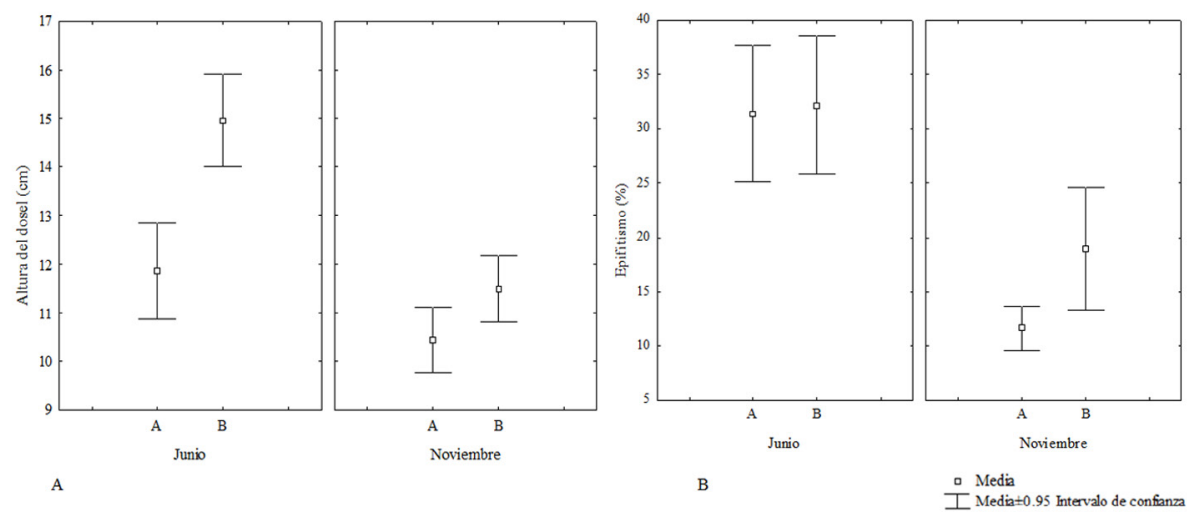

Fig. 3. A. Porcentaje de abundancia relativa de $T$. testudinum entre sitios por meses de muestreo $(\mathrm{F}=2.502 ; P>0.05)$. B. Densidad de vástagos de T. testudinum entre sitios por meses de muestreo (vástagos $\left.\mathrm{m}^{-2}\right)(\mathrm{F}=0.871 ; P>0.05)(\mathrm{A}$ : Las Coloradas. B: El Paso)

Fig. 3. A. Percentage of relative abundance of $T$. testudinum between sampling sites per month. $(\mathrm{F}=2.502, P>0.05)$. B. Shoot density of $T$. testudinum (shoot $\mathrm{m}^{-2}$ ) between sites per month $(\mathrm{F}=0.871, P>0.05)$ (A: Las Coloradas, B: El Paso) 

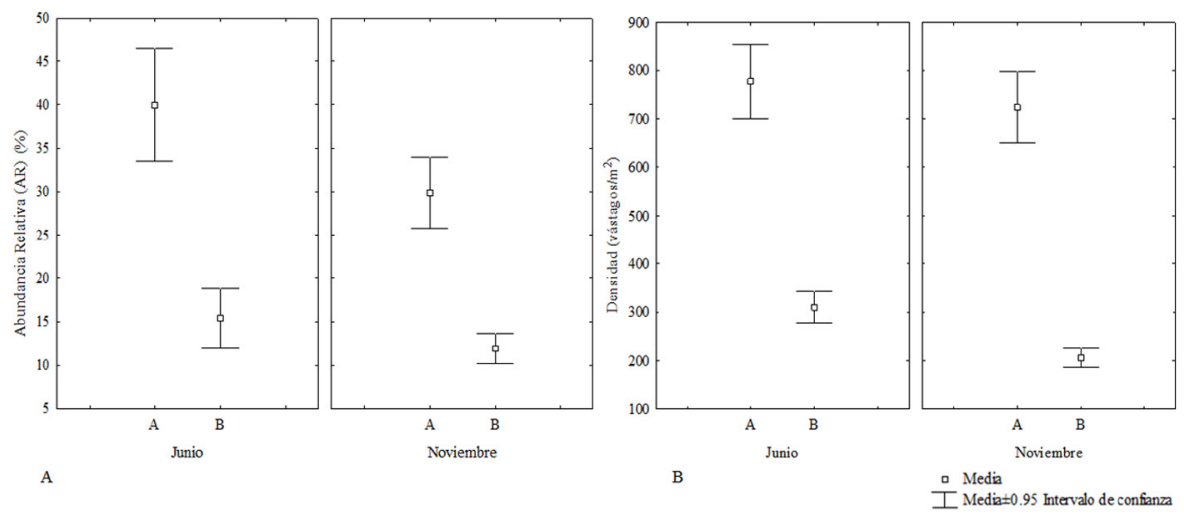

Fig. 4. A. Altura del dosel de T. testudinum (\%) entre sitios por meses de muestreo $(\mathrm{F}=5.995 ; P<0.05)$ B. Porcentaje de epifitismo $(\%)$ de T. testudinum entre sitios por meses de muestreo $(\mathrm{F}=313.147 ; P<0.05)$ (A: Las Coloradas. B: El Paso)

Fig. 4. A. Dossal height of T. testudinum (\%) between sites per month. $(\mathrm{F}=\mathrm{F}=5.995, P<0.05) \mathrm{B}$. Epiphytism percentage $(\%)$ of $T$. testudinum between sampling sites per month $(\mathrm{F}=313.147 ; P<0.05)(\mathrm{A}$ : Site Las Coloradas, B: El Paso)

En Las Coloradas, la altura del dosel de T. testudinum fue menor que para El Paso. Para ambos sitios la altura de $T$. testudinum disminuyó en noviembre $(10.4 \pm 2 \mathrm{~cm}$ y $11.5 \pm 2.1 \mathrm{~cm}$, respectivamente). Según la prueba de ANOVA bifactorial la altura del dosel mostró diferencias significativas entre meses $(\mathrm{F}=34.458 ; P<0.05)$, entre sitios $(\mathrm{F}=24.979 ; P<0.05)$, y entre sitios por meses $(\mathrm{F}=5.995 ; P<0.05)$ (Fig. 4A).

En $T$. testudinum el epifitismo predominante fue de algas rojas costrosas, en las porciones distales de las hojas, las que en su mayoría se observaron necrosadas. En junio, en ambos sitios de estudio, el epifitismo sobre las hojas de T. testudinum fue mayor al $30 \%$, mientras que en noviembre disminuyó hasta el 12\%. El epifitismo fue significativamente diferente entre meses $(\mathrm{F}=38.499 ; P<0.05)$ y entre sitios por meses $(\mathrm{F}=313.147 ; P<0.05)$, pero no entre sitios $(\mathrm{F}=2.369 ; P>0.05)$ (Fig. 4B).

Se lograron identificar un total de 25 especies de macroalgas pertenecientes a 13 géneros distribuidos en 3 Phyla y 6 Grupos Morfofuncionales (GMF) (Cuadro 1). La cobertura promedio de macroalgas, entre los dos sitios muestreados para junio estuvo dominada por el GMF calcáreo, (géneros Halimeda, Penicillus y Udotea, con 
un $7 \% \pm 4.64 \%$ de representación), da por el GMF calcáreo con un 4.5\% seguida de las carnosas (géneros Cau- $\quad \pm 2.76 \%$, seguido del carnoso con un lerpa, Laurencia, con un $4 \% \pm 1.25 \% \quad 4 \% \pm 1.81 \%$. El sitio que más contride representación). En noviembre, buyó a la superioridad porcentual del para ambos sitios, la cobertura pro- GMF calcáreo fue El Paso con un 6\% medio de macroalgas estuvo domina- $\quad \pm 3.55 \%$ de cobertura del grupo.

Cuadro 1. Macroalgas observadas en los pastos marinos de playa Las Coloradas (sitio A) y El Paso (sitio B) para junio y noviembre. Leyenda: Grupo morfofuncional (GMF)

Table 1. Macroalgae observed in the seagrass of Las Coloradas beach (site A) and El Paso beach (site B) during June and November. Text: Morpho-Functional Group (MFG)

\begin{tabular}{|c|c|c|c|c|c|c|}
\hline \multirow{3}{*}{ Phylum } & \multirow{3}{*}{ GMF } & \multirow{3}{*}{ Especie } & \multicolumn{4}{|c|}{ Sitios de muestreos meses } \\
\hline & & & \multicolumn{2}{|c|}{ junio } & \multicolumn{2}{|c|}{ noviembre } \\
\hline & & & $\mathbf{A}$ & B & $\mathbf{A}$ & B \\
\hline \multirow[t]{21}{*}{ Chlorophyta } & verde calcárea & Halimeda incrassata & & $\mathrm{X}$ & $\mathrm{X}$ & \\
\hline & & Halimeda monile & & $X$ & X & \\
\hline & & Halimeda sp. & $\mathrm{X}$ & & & $\mathrm{X}$ \\
\hline & & Penicillus dumetosus & & & $\mathrm{X}$ & \\
\hline & & Penicillus pyriformis & & & $\mathrm{X}$ & \\
\hline & & Penicillus capitatus & $\mathrm{X}$ & $\mathrm{X}$ & & $\mathrm{X}$ \\
\hline & & Rhipocephalus phoenix & & $X$ & & \\
\hline & & Rhipocephalus phoenix f. longifolius & $\mathrm{X}$ & & & $\mathrm{X}$ \\
\hline & & Udotea flabellum & $\mathrm{X}$ & & & $\mathrm{X}$ \\
\hline & & Udotea conglutinata & $\mathrm{X}$ & & & $\mathrm{X}$ \\
\hline & & Avrainvillea nigricans f. parva & $\mathrm{X}$ & & $\mathrm{X}$ & \\
\hline & & Avrainvillea elliottii & $\mathrm{X}$ & & $\mathrm{X}$ & \\
\hline & carnosa & Caulerpa paspaloides & & & $\mathrm{X}$ & $\mathrm{X}$ \\
\hline & & Caulerpa mexicana & & $\mathrm{X}$ & & $\mathrm{X}$ \\
\hline & & Caulerpa cupressoides & & & & $\mathrm{X}$ \\
\hline & & Caulerpa prolifera & & $\mathrm{X}$ & & $\mathrm{X}$ \\
\hline & & Dasycladus vermicularis & & $\mathrm{X}$ & & \\
\hline & foliosa & Microdictyon marinum & $\mathrm{X}$ & & & \\
\hline & globosa & Valonia ventricosa & & $\mathrm{X}$ & $X$ & \\
\hline & & Valonia sp. & $\mathrm{X}$ & & & \\
\hline & filamentosa & Cladophora sp. & & $X$ & & \\
\hline Phaeophyta & foliosa & Dictyota pinnatifida & & $\mathrm{X}$ & & \\
\hline \multirow[t]{2}{*}{ Rhodophyta } & costrosa & Neogoniolithon strictum & $\mathrm{X}$ & & & \\
\hline & carnosa & Laurencia intricata & $\mathrm{X}$ & $\mathrm{X}$ & & \\
\hline
\end{tabular}


Fauna asociada a los pastos marinos hojas de T. testudinum. En playa Las

En los sitios de estudio se identificaron 10 especies de invertebrados pertenecientes a 9 géneros, distribuidos en 6 órdenes y 9 familias, de las cuales 4 pertenecen al Phylum Cnidaria, 3 a Mollusca, 2 a Echinodermata y 1 a Porifera. También fueron observadas ascidias e hidrozoos sobre las

Coloradas se observó mayor diversidad de invertebrados asociados a los pastos marinos que en playa El Paso (Cuadro 2). En noviembre, se observó disminución de la diversidad de invertebrados asociados a las praderas de los sitios de estudio, que persiste solo en el 30\% de las especies.

Cuadro 2. Especies de invertebrados identificados en playa Las Coloradas (sitio A) y El Paso (sitio B), para junio (antes del paso del huracán Irma) y noviembre (luego del paso del huracán Irma), 2017

Table 2. Invertebrate species identified in Las Coloradas beach (site A) and El Paso beach (site B), Cuba, during June (before hurricane Irma) and November (after hurricane Irma), 2017

\begin{tabular}{|c|c|c|c|c|}
\hline \multirow{2}{*}{ Ubicación Taxonómica } & \multicolumn{2}{|c|}{ Junio } & \multicolumn{2}{|c|}{ Noviembre } \\
\hline & $\mathbf{A}$ & $\mathbf{B}$ & $\mathbf{A}$ & $\mathbf{B}$ \\
\hline \multicolumn{5}{|l|}{ Phylum Porifera } \\
\hline \multicolumn{5}{|l|}{ Clase Demospongiae } \\
\hline \multicolumn{5}{|l|}{ Orden Hadromerida } \\
\hline Familia Clionaidae & $\mathrm{X}$ & $\mathrm{X}$ & $\mathrm{X}$ & \\
\hline \multicolumn{5}{|l|}{ Cliona sp. } \\
\hline \multicolumn{5}{|l|}{ Phylum Porifera } \\
\hline \multicolumn{5}{|l|}{ Clase Demospongiae } \\
\hline Orden Hadromerida & $\mathrm{X}$ & & & \\
\hline \multicolumn{5}{|l|}{ Familia Poritidae } \\
\hline Porites porites & $\mathrm{X}$ & $\mathrm{X}$ & & \\
\hline \multicolumn{5}{|l|}{ Porites furcata } \\
\hline \multicolumn{5}{|l|}{ Phylum Cnidaria } \\
\hline \multicolumn{5}{|l|}{ Clase Anthozoa } \\
\hline \multicolumn{5}{|l|}{ Orden Scleractinia } \\
\hline Familia Mussidae & & & $\mathrm{X}$ & \\
\hline
\end{tabular}




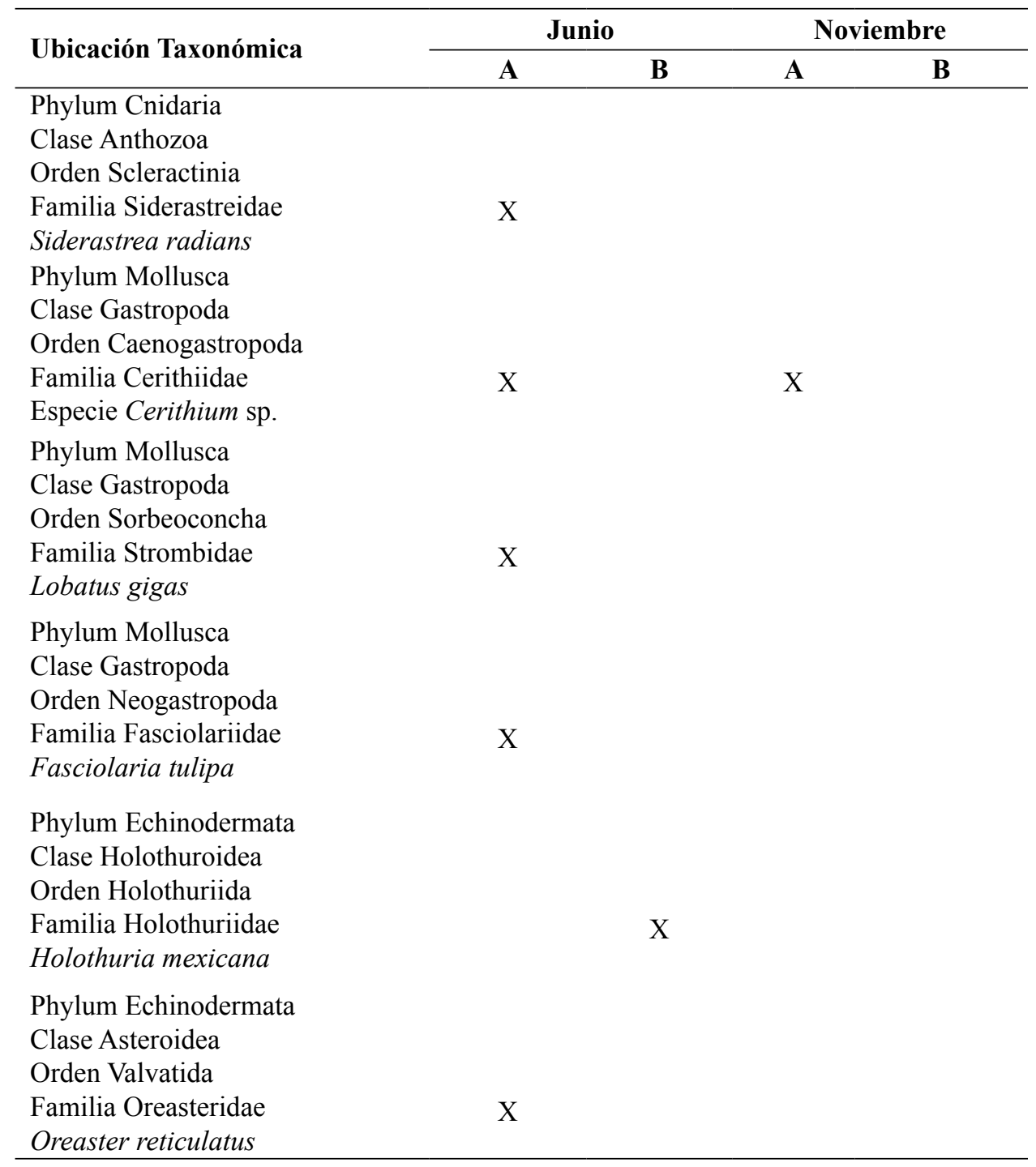

Encuanto a la composición íctica de la especie Halichoeres bivittatus en los pastos marinos, en ambos sitios (Bloch, 1793) (13 individuos) y de estudio, se identificaron 5 especies Gerres cinereus (Walbaum, 1792) (15 pertenecientes a 5 familias y 2 órdenes individuos) (Cuadro 3). En noviembre de la clase Actinopterygii. Se contaron un total de 42 individuos, de los no se observaron peces asociados a los cuales los más representativos fueron pastos marinos de los sitios de estudio. 
Cuadro 3. Peces asociados a los pastos marinos de las playas Las Coloradas y El

Paso, Cuba, en el mes de junio 2017

Table 3. Fish associated to the seagrass in Las Coloradas and El Paso beaches, Cuba, in June 2017

\begin{tabular}{lllc}
\hline Orden & Familia & Especie & N. ${ }^{\circ}$ de individuos \\
\hline \multirow{2}{*}{ Tetraodontiformes } & Tetraodontidae & Sphoeroides spengleri & 4 \\
\multirow{2}{*}{ Perciformes } & Monacanthidae & Monacanthus ciliatus & 2 \\
& Labridae & Halichoeres bivittatus & 13 \\
& Gerreidae & Gerres cinereus & 15 \\
& Carangidae & Caranx ruber & 8 \\
\hline
\end{tabular}

\section{DISCUSIÓN}

Los pastos marinos en playa Las Coloradas y El Paso, pertenecientes a la cayería norte de la provincia de Ciego de Ávila, presentaron cambios en su estructura y composición debido a la incidencia del huracán Irma. Similar resultado registró Guimarais et al. (2013) para los del Parque Nacional Jardines de la Reina, al sureste de Cuba, ante la incidencia del huracán Paloma en 2008. Sin embargo, difiere de lo obtenido por Rodríguez-Ramírez \& Reyes-Nivia (2008) para los pastos marinos en el Caribe colombiano ante las incidencias del huracán Beta en 2005.

La especie T. testudinum fue predominante en ambos sitios de estudio, tanto en junio como en noviembre, lo que coincide con los resultados obtenidos por Martínez-Daranas (2007) para el ASC, ubicado al norte de Cuba, y para otras áreas de la región, como la costa norte de La Habana (Jiménez \& Alcolado, 1989) y Jardines de la Reina
(Bustamante-López et al. 2018) y en general, con lo reportado para el Caribe (van Tussenbroek et al. 2014). Esto corrobora lo planteado por Gallegos et al. (1994), quienes plantean que es la especie clímax en la sucesión de los pastos marinos del Caribe.

El desarrollo de pastos mixtos en playa El Paso (dos angiospermas marinas) y el monoespecífico de $T$. testudinum en playa Las Coloradas coincide con lo registrado por CleroAlonso et al. (2006) para estas zonas, donde registraron a $T$. testudinum como la especie dominante en la pradera, mientras que $S$. filiforme aparecía hacia los bordes de esta.

Enríquez \& Pantoja-Reyes (2005) comprobaron que la mayor parte de la que atenuación de la luz dentro del dosel del pasto es generado por las hojas de la especie $T$. Testudinum; lo que apoya la hipótesis de su capacidad competitiva superior para excluir otras especies de la comunidad macrofitobentónica. En noviembre 
(posterior al huracán Irma), $T$. testudinum permaneció en playa El Paso, mientras que S. filiforme desapareció. Esto podría estar relacionado con que $T$. testudinum tiene un sistema de raíces y rizomas mejor desarrollado que todas las demás especies de pastos marinos en el Atlántico Occidental (van Tussenbroek et al. 2006), lo que determinó que fuera más resistente ante el embate del huracán Irma.

Según Fourqurean \& Rutten (2004), T. testudinum, especie de sucesión tardía, tiene una tasa de crecimiento lento, mayor longevidad, se reproduce casi exclusivamente por propagación vegetativa, y posee estructuras subterráneas que le proporciona mayor fuerza de enterramiento y resistencia a la erosión, lo que le permite persistir pese a la ruptura de sus hojas por alguna perturbación.

Estudios como el de ArellanoMéndez et al. (2011) y Guimarais et al. (2013) relacionados con los efectos de los huracanes no incluyen datos pre-huracán. Sin embargo, Fourqurean \& Rutten (2004) reportan que $S$. filiforme es mucho más susceptible a los estragos ocasionadas por huracanes que $T$. testudinum, y esto es probablemente el resultado de las diferentes estrategias de historia de vida de estas especies. Cruz-Palacio \& van Tussenbroek (2005) determinaron que el sistema radicular de $S$. filiforme es fácilmente desenterrado debido a que posee rizomas delgados y anclados a una profundidad baja, y sus raíces son profundas pero delicadas, por lo que es más sensible que $T$. testudinum a la remoción de sedimentos.

Según Salazar-Vallejo (2002) no ha sido demostrado que la susceptibilidad al oleaje provocada por tormentas o la sedimentación, esté relacionada con la forma de crecimiento de las angiospermas. En Guadalupe, México, las praderas conformadas por Syringodium fueron las más afectadas después del paso del huracán Hugo (Bouchon et al. 1991). Resultados similares fueron reportados por van Tussenbroek et al. (2008) tras el paso del huracán Wilma por Puerto Morelos, México.

En junio, la densidad de $T$. testudinum en playa Las Coloradas (776.9 vástagos $\mathrm{m}^{-2}$ ) fue similar a la reportada por Martínez-Daranas (2007) para el ASC (732.6 vástagos $\left.\mathrm{m}^{-2}\right)$ y a la observada por Reyes (2016) para Santa Lucia (provincia Camagüey) (617.0 vástagos $\mathrm{m}^{-2}$ ), aunque alta, si se compara con lo expuesto por Bustamante- López et al. (2016) para cayo Paredón Grande (provincia de Ciego de Ávila) (entre 48 y 480 vástagos $\mathrm{m}^{-2}$ ). Todas estas zonas ubicadas en la región norte de Cuba.

La densidad de $T$. testudinum en playa Las Coloradas también se puede considerar similar a la obtenida en el estudio realizado por Díaz et al. (2003) sobre los pastos marinos de Colombia, donde se llegó a registrar entre 730 y 985 vástagos $\mathrm{m}^{-2}$. Sin embargo, 
se considera baja con respecto a los resultados obtenidos para los cayos del norte de la provincia de Ciego de Ávila (1 021 vástagos $\mathrm{m}^{-2}$ ) (Clero-Alonso et al. 2006). Por otra parte, la densidad de T. testudinum obtenida en playa El Paso (310.2 vástagos $\mathrm{m}^{-2}$ ) se considera baja con respecto a los sitios anteriormente mencionados, excepto con lo reportado para cayo Paredón Grande (Bustamante- López et al. 2016), cuyos resultados fueron similares. La densidad de vástagos de T. testudinum en los sitios de estudio registraron valores similares a los monitoreados en el nivel del Caribe, a partir del programa CARICOMP (van Tussenbroek et al. 2014).

En este estudio, la densidad mayor se presentó en el sitio Las Coloradas, donde la profundidad fue de $0.6 \mathrm{~m}$, mientras que la menor se presentó en el sitio El Paso con $2.1 \mathrm{~m}$ de profundidad, lo cual coincide con lo expuesto por Gómez-López et al. (2005), quienes plantean que existe una correlación inversamente proporcional entre la densidad de vástagos y la profundidad. En noviembre, tanto la abundancia relativa como la densidad de T. testudinum, a pesar de que fueron menores a las registradas en junio, no mostraron diferencias significativas, por lo que se infiere que el huracán Irma no tuvo una afectación negativa directa sobre estos parámetros ecológicos en los sitios estudiados.

En junio, la altura del dosel de T. testudinum presentó intervalos entre
$11.6 \pm 3.6$ y $14.9 \pm 2.8 \mathrm{~cm}$, similares a la observada por Reyes (2016) para Santa Lucia $(14.9 \mathrm{~cm})$, a los reportados por Bustamante-López et al. (2016) para cayo Paredón Grande $(6-16 \mathrm{~cm})$ y a los registrados para Alta Guajira, Colombia (entre 11 y $19 \mathrm{~cm}$ ) (Gómez-López et al. 2005). No obstante, se consideran bajos al compararlos con los obtenidos por Martínez-Daranas (2007) para el ASC $(20.1 \mathrm{~cm})$, por lo que se pueden catalogar como pastos marinos de poca altura. Por otra parte, la altura del dosel de T. testudinum en los sitios de estudio registraron valores similares a los monitoreados en el nivel del Caribe, a partir del programa CARICOMP (van Tussenbroek et al. 2014).

La baja altura del dosel podría estar relacionada con condiciones de alta visibilidad reportadas por el disco Secchi, lo que garantiza estabilidad al pasto marino, no es necesario, para la planta, realizar esfuerzo energético para crecer, sino solamente para mantenerse o para producir vástagos jóvenes, como plantearan Gómez-López et al. (2005).

En noviembre, la altura de $T$. testudinum disminuyó (entre 1 y $4 \mathrm{~cm}$ ), siendo más significativo para playa El Paso. Esto es uno de los resultados del efecto del huracán Irma, el cual ocasionó enterramiento de vástagos en los sitios estudiados. Según MartínezDaranas et al. (2014), el efecto del oleaje provoca la disminución de la profundidad y el enterramiento de los vástagos. Van Tussenbroek 
(1994) estudió el impacto del huracán Gilberto (septiembre 1988) sobre las praderas de $T$. testudinum en la costa del Caribe mexicano, donde observó reducción en la longitud del vástago, lo que se atribuyó al incremento en la deposición de sedimentos.

En junio el epifitismo estuvo mayormente dado por algas rojas costrosas para playa Las Coloradas y El Paso, con rangos de porcentajes similares a los reportados por Martínez-Daranas (2007) para el ASC (29.6\%). Lo que coincide también con lo registrado para otras zonas del Caribe como la bahía de Cartagena y en las áreas de la Guajira, en Colombia, según Díaz et al. (2003) y Gómez-López et al. (2005). Los valores de porcentaje del epifitismo disminuyeron de forma significativa luego del paso del huracán Irma. Según Borowitzka et al. (2006), el daño físico es un resultado del movimiento del agua y puede incidir de manera negativa en el crecimiento del dosel. Según estos autores las energías de onda alta, experimentadas durante las tormentas de invierno, también eliminan exceso de crecimiento de epífitas que se han acumulado durante los períodos de mayor incremento del verano.

Según Gacia et al. (1999), la comunidad de epífitos es afectada por varios factores que incluyen la temporada climática, los nutrientes y la dinámica de movimiento de la columna de agua; otros autores como Harlin \& Thorne-Miller (1981) y Worm
\& Sommer (2000) hallaron que el porcentaje de epifitismo está directamente relacionado con los niveles de nutrientes, aspecto que no fue medido durante este estudio. Sin embargo, Martínez-Daranas et al. (2007) expuso que en los sitios donde prevalecía T. testudinum, en el ASC, las concentraciones de nutrientes en el agua eran bajas, al compararlas con los valores presentes donde prevalecían otras especies de angiospermas marinas como $S$. filiforme y $H$. wrigthii. Al parecer, los sitios estudiados presentan escasa influencia de las posibles fuentes contaminantes de la isla grande, como planteara Batista-Tamayo et al. (2006), para las aguas de la plataforma exterior de los cayos de dicho archipiélago.

La cobertura de macroalgas fue menor al $6 \%$, lo que se corresponde con lo registrado por Martínez-Daranas (2007) para el 61\% de las estaciones de pastos marinos estudiadas para el ASC; los géneros predominantes (Halimeda, Penicillus, Udotea y Caulerpa) en los pastos marinos de ambos sitios de estudio coinciden con cuatro de los registrados para dicho archipiélago. El predominio de macroalgas del Phylum Chlorophyta en los pastos marinos de ambos sitios fue semejante a lo reportado para el ASC (Martínez-Daranas, 2007), para otros de la plataforma cubana (Suárez et al. 2013; Bustamante-López et al. 2018) y en diferentes partes del Caribe, 
ya que son características de los fondos arenosos (Trilles et al., 2001).

En junio y noviembre para ambos sitios de estudio el grupo de macroalgas mejor representado y con los mayores valores de cobertura fue el GMF calcáreo, resultados similares fueron los obtenidos por Reyes (2016) para playa Santa Lucia, también ubicada en el ASC. La presencia de las macroalgas calcáreas, tanto entre las acompañantes como en las epífitas, indican una estabilidad en el ecosistema al evidenciar que el ecosistema pareciera no estar eutrofizado (Cardoso et al. 2004; Ralph et al. 2006).

En noviembre (luego del paso del huracán Irma), las algas carnosas permanecieron asociadas al pasto marino, principalmente las del género Caulerpa. Esto puede deberse a que estas son dominantes en las lagunas arrecifales con fondos arenosos, pues presentan estructuras de fijación en forma de rizoides o estolones que les permiten adherirse al sedimento, a través de los cuales también pueden absorber nutrientes (Buesa, 1975).

Las especies de corales pétreos identificadas en este estudio se corresponden con tres de las registradas por Barrios et al. (2003) para los pastos marinos de Colombia; Porites porites (Link, 1807), Manicina areolata (Linnaeus, 1758) y Siderastrea radians (Pallas 1766). El hecho de observarse colonias de $M$. areolata desprendidas del sustrato confirma lo planteado por
Ruiz-Zárate et al. (2000): esta especie solo requiere de un sustrato firme en su etapa inicial de crecimiento. Esto también fue observado en junio para la mayoría de las colonias de esta especie identificadas en los sitios de estudio.

Ruiz-Zárate et al. (2000), expone que $M$. areolata utiliza para su fijación, generalmente en los pastos marinos, a especies de algas del género Neogoniolithon. Ello, a pesar de que no pudo ser constatado en este estudio, pudiera ocurrir en los pastos marinos de ambos sitios, pues en playa La Coloradas, donde más colonias de $M$. areolata se observaron, fue donde se identificó la presencia de dicho género de alga.

Claro (2006), expuso que los pastos poco profundos, cercanos a las costas, sirven como zonas de reclutamiento $\mathrm{y}$ refugio a larvas y juveniles de numerosos recursos pesqueros, muchos de ellos de interés económico. Una de las especies de peces que mayor número de individuos aportaron a la ictiofauna asociada a los pastos marinos de ambos sitios fue G. cinereus (mojarra de ley), cuyos juveniles dependen de la presencia de áreas costeras poco profundas como sitios de crianza, dentro de las que se encuentran los pastos marinos (Nagelkerken et al. 2001). También Caranx ruber (Bloch, 1793) (cibi carbonero) reportada como una de las especies de peces asociadas a T. testudinum en los cayos Ratones y Berberia, Puerto Rico (Berrios et al. 2005). 
Los pastos marinos, en playa Las Coloradas y El Paso, presentaron cambios en su estructura y composición debido a la incidencia del huracán Irma, pues según la Estación Meteorológica de primer orden 78 339, CIEC, ubicada en Cayo Coco, antes del huracán se venía arrastrando un proceso de sequía muy intenso, por lo que no se reportó ningún fenómeno sinóptico ni de mesoescala previo al huracán, que pudiera incidir sobre los resultados obtenidos en los sitios estudiados.

\section{CONCLUSIONES}

El huracán Irma fue un evento meteorológico extremo que cambió la composición y estructura de las praderas de pastos marinos en los sitios de playa Las Coloradas y El Paso, al eliminar la especie $S$. filiforme, e incidir, aunque no de forma significativa, en la disminución de la abundancia relativa y densidad de T. testudinum, y sí significativamente en la altura del dosel y el porcentaje de epifitismo de esta especie, así como en la disminución notoria de la fauna asociada. Se debe continuar el monitoreo de los sitios de pastos marinos de playa Las Coloradas y El Paso, con el fin de estudiar su nivel de recuperación en el tiempo.

\section{AGRADECIMIENTOS}

Las autoras agradecen a Francisco Salmón Moret y Felipe Matos Pupo, del Centro de Investigaciones de Ecosistemas Costeros (CIEC), Cayo Coco. A Evelio A. Alemán Martín de la Marina Marlin Azulmar del Parque Nacional Jardines de la Reina, a Roberto González de Zayas, del Centro de Estudios Geomáticos, Ambientales y Marinos (GEOMAR), México y, a los evaluadores de la Revista Ciencias Marinas y Costeras por sus valiosas sugerencias.

\section{BIBLIOGRAFÍA}

Alcolado, P. M., Menéndez, G., García-Parrado, P., Zúñiga, D., Martínez-Daranas, B., Sosa M. \& Gómez R. (1998). CARICOMP-Caribbean coral reef, seagrass and mangrove sites. Cayo Coco, Sabana-Camaguey Archipelago, Cuba. La Habana, Cuba: CARICOMP.

Arellano-Méndez, L. U., Liceaga-Correa, M. A., Herrera-Silveira, J. A. \& Hernández-Núñez, H. (2011). Impacto por huracanes en las praderas de Thalassia testudinum (Hydrocharitaceae) en el Caribe Mexicano. Rev. Biol. Trop., 59(1), 385-401.

Barrios, L. M., Gómez-López, D. I. \& Montoya-Maya, P. (2003). Estructura de la comunidad biológica asociada a las praderas en Colombia. En J. M. Díaz (Ed.). Las praderas de pastos marinos en Colombia: Estructura y distribución de un ecosistema estratégico (pp. 81-111). Santa Marta, Colombia. Serie Publicaciones Especiales. INVEMAR. 
Batista-Tamayo, L. M., González, R., Zúñiga, A., Matos, F., Hernández, L., \& González, D. (2006). Atributos físicos del norte de la provincia Ciego de Ávila. En CIEC (Ed.) Ecosistemas costeros: biodiversidad y gestión de recursos naturales (pp. 1-76). Compilación por el XV Aniversario del CIEC. CITMA, Cuba.

Berrios, J. M., González- Azar, J. K. \& Díaz-Rodríguez, I. (2005). Fish Population Studies of the Seagrass Beds and Coral Reefs of Cayo Berberia and Cayo Ratones, Ponce, PR. Florida, EE. UU.: NOAA/RSMAS.

Borowitzka, M. A., Lavery, P. \& Keulen, M. V. (2006). Epiphytes of seagrasses, In A. W. D. Larkum, R. J. Orth \& C. M. Duarte (Eds.), Seagrasses: Biology, Ecology and Conservation, (pp. 441461). Dordrecht, The Netherlands: Springer.

Borum, J., Duarte, C. M., Krause-Jensen, D. \& Greve, T. (2004). European seagrasses: an introduction to monitoring and management. Copenhagen, Denmark: The M\&MS Project.

Bouchon, C., Bouchon-Navaro, Y., Imbert, D. \& Louis, M. (1991). The effect of hurricane Hugo on the coastal environment of Guadeloupe Island (FWI). Ann. Inst. Oceanogr., 67(1), 5-33.

Brock, V. E. (1954). A preliminary report on a method of estimating reef fish populations. J. Wildl. Manage., 18(3), 297308. https://doi.org/10.2307/3797016

Buesa, R. (1975). Population biomass and metabolic rates of marine angiosperms on the northwestern Cuban shelf. Aquat. Bot., 1, 11-23.

Bustamante-López, C., Hernández-Fernández, L., Pina-Amargós, F. (2016). Caracterización de los pastos marinos de Paredón Grande, norte de la provincia de Ciego de Ávila, Cuba. Rev. Inv. Mar., 35(2), 74-90.
Bustamante-López, C., Hernández-Fernández, L., González de Zayas, R., Dulce- Sotolongo, L. \& Pina-Amargós, F. (2018). Pastos marinos de Pasa Caballones, Parque Nacional Jardines de la Reina, Cuba. Rev. Inv. Mar., 38(2), 28-44.

Cardoso, P. G., Pardal, M. A., Lillebo, A. I., Ferreira, S. M., Raffaelli, D. \& Marques, J. C. (2004). Dynamic changes in seagrass assemblages under eutrophication and implications for recovery. Exp. Mar. Biol. Ecol., 302(2), 233-248.

Claro, R. (2006). Conservación y Manejo. En

R. Claro (Ed). La Biodiversidad marina de Cuba. La Habana, Cuba: Instituto de Oceanología, Ministerio de Ciencia, Tecnología y Medio Ambiente.

Claro, R., Cantelar-Ramos, K., Pina-Amargós, F. \& García-Arteaga, J. P. (2000). Biodiversidad y manejo de la ictiofauna del archipiélago-Sabana-Camagüey. La Habana. Cuba: Instituto de Oceanología.

Claro, R. \& Garcia-Arteaga, J. P. (1994). Estructura de las comunidades de peces en los arrecifes del grupo insular Sabana- Camagüey, Cuba. Avicennia, 2, 83-107.

Clero-Alonso, L., Pina-Amargós, F., Hernández-Fernández, L., Martín-Blanco, F., Zúñiga-Ríos, D., Cowling S., Brady, A. K. \& Caldwell, S. (2006). Biota acuática del norte de la provincia de Ciego de Ávila. En CIEC (Ed.), Ecosistemas Costeros: biodiversidad y gestión de recursos naturales. Compilación por el XV Aniversario del CIEC (pp. 182205). Habana, Cuba. CITMA.

Cruz-Palacio, V. \& van Tussenbroek, B. I. (2005). Simulation of hurricane-like disturbances on a Caribbean seagrass bed. J. Exp. Mar. Biol. Ecol. 324(1), 44-60. 
Dawes, C. J. \& Mathieson, A. C. (2008). The Seaweeds of Florida. Gainesville, EE. UU.: University Press of Florida.

den Hartog, C. \& Kuo, J. (2006). Taxonomy and biogeography of seagrasses. En A. W. D. Larkum, R. J. Orth \& C. M. Duarte (Eds.). Seagrass: biology, ecology and conservation. (pp. 1-23). Dordrecht, The Netherlands.: Springer.

de la Torre-Núñez, E., Rodiero-Guerra, I., Menéndez, R. \& Pérez-Carrasco, D. (2012). Thalassia testudinum, una planta marina con potencialidades de uso terapéutico. Rev. Cub. Plantas Medicinales. 17(3), 288-296.

Díaz, L., Barrios, M. \& Gómez-López, D. I. (2003). Las praderas de pastos marinos en Colombia: Estructura y distribución de un ecosistema estratégico. Serie Publicaciones Especiales. Santa Marta, Colombia: INVEMAR.

Duarte, C. M. \& Sand-Jensen, K. (1990). Seagrass colonization: biomass development and shoot demogrphy in $C y$ modocea nodosa patches. Mar. Ecol. Prog. Ser., 67(1), 97-103.

Enríquez, S. \& Pantoja-Reyes, N. I. (2005). Form-function analysis if the effect of canopy morphology on leaf self-shading in the seagrass Thalassia testudinum. Oecologia, 145(2), 235-243.

Fourqurean, J. W. \& Rutten, L. M. (2004). The impact of Hurricane Georges on softbottom, back reef communities: Site-and species-specific effects in South Florida seagrass beds. Bull. Mar. Sci., 75(2), 239-257.

Gacia, E., Littler, M. M. \& Littler, D. S. (1999). An experimental text of the capacity of food web interactions (fish-epiphytes-seagrasses) to offset the negative consequences of eutrophication on seagrass communities. Estuar. Coast. Shelf Sci., 48(6), 757-766.
Gallegos, M. E., Merino, M., Rodríguez, A., Marbá, N. \& Duarte, C. M. (1994). Growth patterns and demography of pioneer Caribbean seagrasses Halodule wrightii and Syringodium filiforme. Mar. Ecol. Prog. Ser., 109, 99-104.

Gómez-López, D., Duque, G. \& Garzón, P. A. (2005). Estructura vegetal y productividad foliar de praderas de Thalassia testudinum (Banks ex König) en el departamento de La Guajira, Caribe Colombiano. En J. C. Narváez (Ed.), Informe del estado de los ambientes marinos y costeros en Colombia (pp. 147-156). Santa Marta, Colombia: INVEMAR.

González-Ferrer, S. (2009). Diversidad de Organismos. Celenterados-Filo Cnidaria: Clase Anthozoa, Subclase Zoantharia, corales pétreos Orden Scleractinia. En R. Claro (Ed.), Biodiversidad marina de Cuba (pp. 42-46). La Habana, Cuba: Instituto de Oceanología.

Guimarais, M., Zúñiga, A., Pina, F. \& Matos, F. (2013). Efectos del Huracán Paloma sobre los pastos marinos del archipiélago Jardines de la Reina, Cuba. Rev. Biol. Trop., 61(3), 1425-1432. https:// doi.org/10.15517/rbt.v61i3.11969

Guiry, M. D. \& Guiry, G. M. (2018). AlgaeBase. World-wide electronic publication. National University of Ireland, Galway. Recuperado en abril 30, 2017, disponible en http://www.algaebase.org.

Harlin, M. M. \& Thorne-Miller, B. (1981). Nutrient enrichment of sea grass beds in a Rhode Island coastal lagoon. Mar. Biol., 65(3), 221-229.

Hemminga, M. A. \& Duarte, C. M. (2000). Seagrass Ecology. New York, EE. UU.: University of Cambridge.

Humann, P. (2002). Reef Creature Identification. Jacksonville, Florida, EE.UU.: New World Publications, Inc.Humann, P. \& Deloach, N. (2006). Reef fish 
identification, Florida Caribbean Bahamas. Jacksonville, Florida, EE. UU.: New World Publications, Inc.

IPCC. (2007). Fourth Assessment Report of the Intergovernmental Panel on Climate Change. Cambridge, United Kingdom and New York, EE. UU.: Cambridge University Press.

Jiménez, C. \& Alcolado, P. M. (1989). Comportamiento estacional de la biomasa vegetal en un seibadal de Cuba. Acta Bot. Cub., 71, 1-10.

Littler, D. S. \& Littler, M. M. (2000). Caribbean Reef Plants. An Identification Guide to the Reef Plants of the Caribbean, Bahamas, Florida and Gulf of Mexico. Washington D. C, EE. UU.: Offshore Graphics.

Littler, D. S., Littler, M. M. \& Hanisak, M. D. (2008). Submersed plants of the Indian River Lagoon. Florida, EE. UU.: Offshore Graphics Washington.Marbà, N. \& Duarte, C. M. (1994). Growth response of the seagrass Cymodocea nodosa to experimental burial and erosion. Mar. Ecol. Prog. Ser., 107, 307311.

Martínez-Daranas, B. (2007). Características y estado de conservación de los pastos marinos en áreas de interés del Archipiélago Sabana-Camagüey, Cuba. Tesis de doctorado no publicada. Universidad de La Habana. Cuidad de La Habana, Cuba.

Martínez-Daranas, B. (2010). Los pastos marinos de Cuba y el cambio climático. En A. Hernández-Zanuy \& P. M. Alcolado (Eds). La biodiversidad en ecosistemas marinos y costeros del litoral de Iberoamérica y el cambio climático: I. Memorias del Primer Taller de la RED CYTED BIODIVMAR, La Habana, Cuba.
Martínez-Daranas, B. (2014). Protejamos los pastos marinos. Flora y Fauna, 18(2), 8-11.

Martínez-Daranas, B., Cano Mallo, M. \& Clero- Alonso, L. (2009). Los pastos marinos de Cuba: Estado de conservación y manejo. Oceanológica, 5(5), 24-44.

Martínez-Daranas, B., Cano-Mallo, M., Perdomo, M. E., Clero-Alonso, L., Díaz-Larrea, J., Guimaraes, M., Zúñiga-Ríos, D., Alcolado, P. M., Duarte-Quesada, C. \& Siret, S. (2007). Estado de los ecosistemas marinos y costeros, y algunas características ambientales $\mathrm{y}$ tendencias. Estado de los pastos marinos. En P. M. Alcolado, E. E. García \& M. Arellano-Acosta (Eds.). Ecosistema Sabana-Camagüey. Estado actual, avances y desafios en la protección y uso sostenibles de la biodiversidad (pp. 51-56). La Habana, Cuba: Editorial Academia.

Martínez-Daranas, B., Hernández-Ávila, A. \& Valdez-Pérez, A. (2014). Ecosistemas Prioritarios. Resultados del programa de pastos marinos. En A. Hernández-Ávila (Ed.). Estado actual de la Biodiversidad Marina Costera, en la región de los archipiélagos del Sur de Cuba (pp. 51-58). Ministerio de Ciencia, Tecnología y Medio Ambiente. La Habana, Cuba: Impresos Dominicanos s. r. 1.

Martínez-Daranas, B., Macías Reyes, D. \& Cano Mallo, M. (2013). Protocolo para el muestreo de los pastos marinos. La Habana, Cuba: Centro Nacional de Áreas Protegidas.

Nagelkerken, S., Kleijnen, T., Klop, R. A., van den Brand, C. J., Cocheret de la Morinière, E. \& van dervelde, G. (2001). Dependence of Caribbean reef fishes on mangroves and seagrass beds as nursery habitats: a comparison of fish faunas between bays with and without 
mangroves/seagrass beds. Mar. Ecol. Prog. Ser., 214, 225-235.

Nordlund, L. M., Jackson, E. L., Nakaoka, M., Samper-Villarreal, J., Beca-Carretero, P. \& Creed, J. C. (2018). Seagrass ecosystem services - What's next? Mar. Pol. Bul., 134, 145-151. https://doi.org/10.1016/j.marpolbul.2017.09.014

Ralph, P. J., Tomasko, D. A., Moore, K., Seddon, S. \& Macinnis-Ng, C. M. O. (2006). Human impacts on seagrass: Eutrophication, sedimentation and contamination. En A. W. D. Larkum, R. J. Orth \& C. M. Duarte (Eds.). Seagrasses: Biology, Ecology and Conservation (pp. 567-593). Dordrecht, The Netherlands: Springer.

Reyes, L. M. (2016). Distribución y conservación de los pastos marinos en la playa Santa Lucía, Camagüey, Cuba. Tesis de maestría no publicada. Centro de Investigaciones Marinas: Universidad de La Habana, Cuba.

Ridler, M. S., Dent, R. C. \& Arrington, D. A. (2006). Effects of Two Hurricanes on Syringodium filiforme, Manatee Grass, Within the Loxahatchee River Estuary, Southeast Florida. Est. and Coast., 29, 1019-1025. https://doi.org/10.1007/ BF02798664

Rodríguez-Ramírez, A. \& Reyes-Nivia, M. C. (2008). Evaluación rápida de los efectos del huracán Beta en la Isla de Providencia (Caribe colombiano). Bol. Invest. Mar. Cost., 37(1), 217-224.

Rodríguez-Ramírez, A., Reyes-Nivia, M. C., Navas-Camacho, R., Vega-Sequeda, J., Olaya, J., Duque, G., Garzón-Ferreira, J., Zapata, F. \& Orozco, C. (2005). Estado de los ecosistemas marinos y costeros. En J. C. Narváez (Ed.), Informe del Estado de los Ambientes Marinos y Costeros en Colombia (pp. 71-134). Santa Marta, Colombia: INVEMAR.
Ruiz-Zárate, M., Espinosa-Avalos, J., Carricart-Ganivet, J. P. \& Fragoso, D. (2000). Relationships between Manicina areolata (Cnidaria: Scleractinia), Thalassia testudinum (Anthophyta) and Neogoniolithon sp. (Rhodophyta). Mar. Ecol. Prog. Ser., 206, 135-146. https://doi.org/10.3354/meps206135

Salazar-Vallejo, S. I. (2002). Huracanes y biodiversidad costera tropical. Rev. Biol. Trop., 50(2), 415-428,

Spalding, M., Taylor, M. Ravilious, C., Short, F. \& Green, E. (2003). The distribution and status of seagrasses. World atlas of seagrasses. Berkeley, EE. UU.: University of California Press.

StatSoft, Inc. (2004). STATISTICA 7.0 for Windows. Tulsa, Oklahoma, EE. UU.: StatSoft.

Suárez, A. M., Martínez-Daranas, B., Guimarais-Bermejo, M. \& Volta, R. (2013). Macroalgas del Golfo de Ana María, SE de Cuba. Rev. Invest. Mar., 33(2), $1-6$.

Suárez, A. M., Martínez-Daranas, B. \& Alfonso, Y. (2015). Macroalgas marinas de Cuba. La Habana, Cuba.: Editorial UH.

Trelles, J., Suárez, A. M. \& de la Guardia, E. (2001). Macroalgas dominantes de Playa Herradura, plataforma noroccidental de Cuba: Caulerpales y Dictyotales. Rev. Invest. Mar., 22(1):1-6,

Valdés-Montero, J. F., Casanueva-Ayala, R. \& González-Baragaño, J. A. (2000). Diccionario Geográfico de Cuba. Oficina de Hidrografia y Geodesia. Comisión Nacional de Nombres Geográficos. La Habana, Cuba.

Vales, M., Álvarez, A., Montes, L., Ávila, A. (1998). Estudio nacional sobre la diversidad biológica en la República de Cuba. Programa de Naciones Unidas para el Medio Ambiente. Centro Nacional de Biodiversidad. Instituto de 
Ecología y Sistemática. CITMA, La Habana, Cuba.

van Tussenbroek, B. I. (1994). The impact of hurricane Gilbert on the vegetative development of Thalassia testudinum in Puerto Morelos coral reef lagoon, Mexico: a retrospective study. Bot. Mar., 37(5), 421-428.

van Tussenbroek, B. I., Vonk, J. A., Stapel, J., Erftemeijer, P. L. A., Middelburg, J. J. \& Zieman, J. C. (2006). The biology of Thalassia: Paradigms and recent advances in research. En A. W. D. Larkum, R. J. Orth, \& C. M. Duarte (Eds.). Seagrasses: Biology, Ecology and Conservation (pp. 409-439). Dordrecht, The Netherlands: Springer. van Tussenbroek, B. I., Barba Santos, M. G., Vandijk, J. K., Alcaraz, S. N. \& Calderon, M. (2008). Selective elimination of rooted plants from a tropical seagrass bed in a back-reef lagoon: a hypothesis tested by Hurricane Wilma (2005). J. Coast. Res., 24(241), 278-281.

van Tussenbroek, B. I., Corte's, J., Collin, R., Fonseca, A. C. \& Gayle, P. M. H. (2014). Caribbean-Wide, Long- Term Study Of Seagrass Beds Reveals Local Variations, Shifts in Community Structure and Ocasional Collapse. Plos One. 9(3), e90600.

Worm, B. \& Sommer, U. (2000). Rapid direct and indirect effects of a singlenutrient pulse in a seaweed-epiphyte-grazer system. Mar. Ecol. Prog. Ser., 202, 283-288. 